\title{
Simulation-based learning of transesophageal echocardiography in cardiothoracic surgical trainees: A prospective, randomized study
}

\author{
Jeremy Smelt, MRCS, ${ }^{\mathrm{a}}$ Carlos Corredor, FRCA, ${ }^{\mathrm{b}}$ Mark Edsell, FRCA, ${ }^{\mathrm{b}}$ Nick Fletcher, FRCA, \\ Marjan Jahangiri, FRCS, ${ }^{\mathrm{a}}$ and Vivek Sharma, FRCA ${ }^{\mathrm{b}}$
}

\begin{abstract}
Objectives: The Intercollegiate Surgical Curriculum now recommends that cardiac surgical trainees should be able to understand and interpret transesophageal echocardiography images. However, cardiac surgical trainees receive limited formal transesophageal echocardiography training. The objective of this study was to assess the impact of simulation-based teaching versus more traditional operating room teaching on transesophageal echocardiography knowledge in cardiac surgical trainees.
\end{abstract}

Methods: A total of 25 cardiac surgical trainees with no formal transesophageal echocardiography learning experience were randomly assigned by computer to a study group receiving simulation-based transesophageal echocardiography teaching via the Heartworks (Inventive Medical, London, UK) simulator $(\mathrm{n}=12)$ or a control group receiving transesophageal echocardiography teaching during elective cardiac surgery $(\mathrm{n}=13)$. Each subject undertook a video-based test composed of 20 multiple choice questions on standard transesophageal echocardiography views before and after teaching.

Results: There was no significant difference in the pretest scores between the 2 groups $(P=.89)$. After transesophageal echocardiography teaching, subjects within each group demonstrated a statistically significant improvement in transesophageal echocardiography knowledge. Although the subjects within the simulation group outperformed their counterparts in the operating room teaching group in the post-test scores, this difference was not significant $(P=.14)$.

Conclusions: Despite the familiarity with transesophageal echocardiography images during surgery, subjects in the simulation group performed at least as well as those in the operating room group. Surgical trainees will benefit from formal transesophageal echocardiography teaching incorporated into their training via either learning method. (J Thorac Cardiovasc Surg 2015;150:22-5)

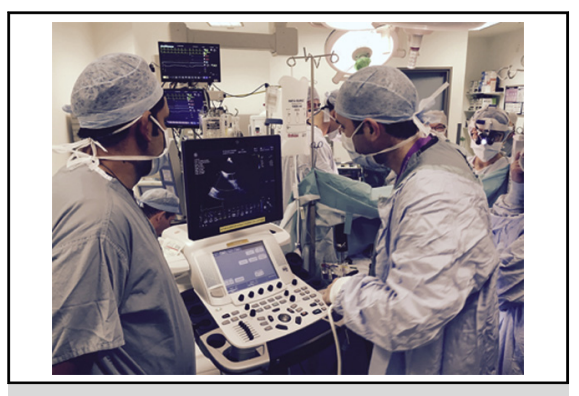

One of the faculty demonstrating TEE views in the operating room

\section{Central Message}

Simulated and operating room teaching of TEE should be incorporated into cardiothoracic surgical training.

\section{Perspective}

This study highlights the beneficial impact of 2 teaching methods, intraoperative and simulated, on the ability of cardiac surgical trainees to understand basic cardiac anatomy using TEE. We recommend the inclusion of formal TEE learning via either method into the cardiac surgical curriculum to improve knowledge and facilitate joint decision-making, which may affect patient outcomes.

See Editorial Commentary page 26.
Transesophageal echocardiography (TEE) is an essential modality within cardiac surgery. Its use perioperatively is paramount in ensuring accurate surgical planning and demonstrating adequate surgical results. The role of TEE has expanded into perioperative management of patients in other noncardiac surgical specialties and management of critically ill patients in the intensive care unit. ${ }^{1-4}$

From the ${ }^{\mathrm{a}}$ Department of Cardiothoracic Surgery and ${ }^{\mathrm{b}}$ Cardiothoracic Anesthesia, St George's Hospital, London, United Kingdom.

Received for publication Dec 24, 2014; revisions received April 7, 2015; accepted for publication April 16, 2015; available ahead of print May 16, 2015

Address for reprints: Marjan Jahangiri, FRCS, Department of Cardiothoracic Surgery,

St George's Hospital, Blackshaw Rd, London SW17 0QT (E-mail: marjan. jahangiri@stgeorges.nhs.uk).

$0022-5223 / \$ 36.00$

Copyright (c) 2015 by The American Association for Thoracic Surgery

http://dx.doi.org/10.1016/j.jtcvs.2015.04.032
Guidelines have been published outlining the importance of TEE and recommending its use in both cardiac and noncardiac surgery. 5,6

The Intercollegiate Surgical Curriculum recommends that cardiac surgical trainees should be able to understand and interpret TEE images. ${ }^{7}$ However, cardiac surgical trainees receive limited formal TEE training, if any. The London regional teaching program, which has the highest proportion of trainees, has not included teaching on TEE. This is thought to reflect national practice. There are accredited courses available for TEE learning, but these tend to be attended mainly by critical care and anesthesiologists.

The importance of becoming proficient in the use of TEE is well recognized in anesthesia, and accreditation has been developed to support this. ${ }^{8}$ The traditional approach for 


\section{Abbreviation and Acronym \\ $\mathrm{TEE}=$ transesophageal echocardiography}

TEE training has been self-directed learning via literature and a lecture-based approach. The intraoperative "handson" approach, which may be regarded as the gold standard, can be difficult to achieve for trainees because of reduced working hours, shift patterns, service provision priorities, and the unpredictability of operating lists and intraoperative situations. Thus, methods of learning this skill in a more time effective manner have been analyzed. ${ }^{9}$

The role and benefit of simulation in medical education have been established. ${ }^{10}$ Previous studies have highlighted the impact of simulation-based echocardiography learning on echo-naïve anesthesia and cardiology trainees. ${ }^{11-16}$ Because of the limited formal training opportunities in TEE available to cardiac surgical trainees, the role of TEE learning via conventional and simulation-based methods have not been previously studied.

The aim of this study was to assess the impact of simulation-based teaching versus more traditional operating room teaching on TEE knowledge in cardiac surgical trainees.

\section{MATERIALS AND METHODS}

Approval for the study from the joint research and enterprise office was obtained. Each subject undertook a video-based test composed of 20 multiple choice questions on cardiac anatomy associated with the 20 standard TEE views before teaching. This was designed by a TEE-accredited anesthetist who was blinded to all aspects of the study. Abnormal anatomy, pathology, and other echocardiography modalities such as Doppler were not assessed as part of this study. A correct answer scored 1 mark, with an incorrect answer scoring zero. There was no negative marking.

To validate the questionnaires, the same video-based tests were administered to cardiac anesthesia consultants accredited in the perioperative use of TEE. The consultants scored a mean (standard deviation) of $92 \%(5 \%)$ and $90 \%(3 \%)$ in the pre- and postintervention tests, respectively.

The subjects were then randomly assigned via computer-based software to a study group receiving simulation-based TEE teaching (Heartworks, Inventive Medical, London, $\mathrm{UK})(\mathrm{n}=12)$ or a control group receiving intraoperative TEE teaching during elective cardiac surgery $(n=13)$. The simulator consists of the head and torso of a mannequin, within whose mouth you can place and manipulate a TEE probe. The images, which are dependent on the position of the probe in the mannequin, are then displayed on a computer screen. The simulation group was shown the standard TEE views on the simulator, as we previously described. ${ }^{9}$ Trainees were allowed multiple attempts at identifying the cardiac sono-anatomy associated with the 20-standard TEE views until they were satisfied. The operating room group was shown the 20-standard TEE views on an anesthetized patient undergoing elective coronary artery bypass graft surgery before the use of diathermy or establishing cardiopulmonary bypass. The images were recorded, and the subjects were able to review these images multiple times in a manner similar to their simulation-based learning counterparts. The trainees in the operating group were extras. Their only task was to be involved in the teaching session.
Both groups were taught the sequential TEE examination taken from the American Society of Anesthesiologists/Society of Cardiovascular Anesthesiologists guidelines. ${ }^{17}$ Both groups were taught the basic cardiac anatomy during their session and were demonstrated how to obtain the standard views of the heart by a TEE-accredited anesthetist. Each session lasted the same amount of time ( 2 hours). The objective of both sessions was to enable the subjects to identify the cardiac anatomy associated with the 20-standard TEE views. Trainees were asked not to seek out additional TEE learning material during the day.

At the end of the training day ( $\sim 4$ hours after the conclusion of TEE teaching), each subject then undertook a second video-based test composed of 20 multiple choice questions on standard TEE views. This postintervention test was of the same format as the preintervention test but composed of different questions.

\section{Statistical Analysis}

Statistical analysis involved the test of normality, after which the Wilcoxon signed-rank test was used to compare results within the groups and the Mann-Whitney $U$ test was used to compare results between the groups. Statistical analyses were performed using SPSS 19.0 (SPSS Inc, Chicago, Ill).

\section{RESULTS}

All 25 subjects who participated in the study and received TEE learning via either method undertook both the pre- and postintervention tests. There was no significant difference in the pretest scores between the 2 groups $(P=.89)$. After TEE teaching, subjects within each group demonstrated a statistically significant improvement in TEE knowledge. Although the subjects within the simulation group outperformed their counterparts in the operating roombased teaching group in the post-test scores, this difference was not significant $(P=.14)$. Table 1 summarizes the scores of the pre- and postintervention tests in both arms of the study.

\section{DISCUSSION}

This study illustrates the beneficial effect of formal TEE learning on the ability of cardiac surgical trainees to identify cardiac sono-anatomy associated with the 20 -standard TEE views.

The utility and impact of simulation-based TEE learning in anesthesia trainees have been studied by several investigators. Bose and colleagues ${ }^{13}$ compared the efficacy of simulation-based TEE learning with conventional methods, such as textbooks, articles, and web-based resources. The authors found a statistically significantly higher change in scores between the pre- and postintervention tests in the simulation group (median [interquartile range]) (28 [16-52]) when compared with the conventional teaching group $(1[9-35]) ; P=.007 .{ }^{13}$ Ferrero and co-workers ${ }^{16}$ assessed the effect of simulation-based training versus traditional didactic teaching on the ability of 42 anesthesia trainees to acquire TEE images. The authors found that $71 \%$ of the images acquired by the trainees in this simulator group were considered acceptable for clinical use compared with $48 \%$ in the traditional 
TABLE 1. Multiple choice question scores before and after teaching

\begin{tabular}{lccc}
\hline & $\begin{array}{c}\text { Operating room } \\
\text { group }(\mathbf{n}=\mathbf{1 3}) \\
\text { Mean \% (SD) }\end{array}$ & $\begin{array}{c}\text { Simulation } \\
\text { group }(\mathbf{n}=\mathbf{1 2}) \\
\text { Mean \% (SD) }\end{array}$ & $\begin{array}{c}\boldsymbol{P} \text { value } \\
\text { (between } \\
\text { groups) }\end{array}$ \\
\hline Preteaching score & $41.6(2.3)$ & $42.2(2)$ & .89 \\
Post-teaching score & $70.4(2)$ & $76(1.4)$ & .14 \\
$P$ value (within group) & $<.001$ & $<.001$ & \\
\hline
\end{tabular}

$S D$, Standard deviation

teaching group. ${ }^{16}$ Our study did not compare performance of image acquisition skills gained as a result of either teaching method because understanding TEE images and identifying cardiac sono-anatomy associated with the 20-standard TEE images are deemed more relevant in our study population. More recently, Ogilvie and colleagues ${ }^{18}$ found a statistically significant increase in the postintervention test scores of echo-naïve anesthesia and medical trainees after TEE teaching by both intraoperative and simulation-based methods. However, they also reported a statistically significant difference in the postintervention test scores between the trainees taught via simulation and conventional methods and the trainees in the simulation cohort outperforming their counterparts who received operating room-based teaching. ${ }^{18}$ Although we found a statistically significant increase in the postintervention test scores within both groups of surgical trainees, our study could not demonstrate a statistically significant benefit of simulation over operating room-based TEE learning in cardiac surgical trainees.

Our findings may lead one to infer that contrary to the findings of the aforementioned TEE studies, there is no perceived benefit of simulation-based TEE learning over operating room-based TEE learning in cardiac surgical trainees. We believe that an alternate explanation exists. The cohort of trainees who participated in our study were cardiac surgical trainees of different levels who did not have any formal experience of TEE learning, but may be accustomed to looking at TEE images in the perioperative period. All trainees were from the same training program (ie, following the same curriculum). Both groups had equal levels of expertise. Although this would induce a bias in the ability of these trainees to identify cardiac sono-anatomy, we believe that the impact of this bias would be evident in the preintervention test scores and would be distributed evenly in both groups.

Cardiac surgical trainees wanting to acquire knowledge of TEE face a dilemma. Although the operating room seems to be the most obvious choice, the priority and expectation of surgical trainees are often to assist with the surgical procedure itself rather than concentrate on TEE images. Furthermore, intraoperative time constraints, operating room scheduling patterns, and the advent of the European Working Time Directive also limit the TEE learning opportunities that otherwise would be available.
Simulation-based TEE learning addresses these issues by providing a scheduled protected learning environment, high-quality images, and no patient interaction, thereby minimizing risk.

\section{Study Limitations}

Cardiac surgical trainees are not truly echo-naïve, and the issue of bias has been discussed earlier. Also, the interpretation and generalization of these results to a wider population are restricted by the small sample size. The number of the subjects in the study is too small to detect a significant difference between the 2 methods of learning TEE; therefore, only a weak trend was seen in favor of simulation training. Because the postintervention test was conducted within 4 hours of TEE learning, it could be argued that the knowledge acquired during the study day would be attributed to short-term memory or immediate recall and would not be able to indicate any benefit in the long-term. Our study demonstrates improvement in recognition of only the most basic cardiac sono-anatomy. Advanced TEE skills have not been assessed in this study. The benefit of a "one only" teaching method will decrease over time in the absence of regular reinforcement. Finally, the improvement in the postintervention test scores in our subjects should be interpreted only as improved understanding of basic cardiac sono-anatomy associated with the 20 -standard views and a small step toward echo-competence.

\section{CONCLUSIONS}

Our study shows that despite the familiarity of cardiac surgical trainees with intraoperative TEE images, subjects in the simulation group performed at least as well as those in the operating room group. The study highlights the impact of both teaching methods on the ability of cardiac surgical trainees to understand basic cardiac anatomy associated with the standard TEE views. We recommend the inclusion of formal TEE learning via either method into the cardiac surgical curriculum with an aim to improve perioperative TEE knowledge and facilitate joint decision-making that may affect patient outcomes.

\section{Conflict of Interest Statement}

N.F. is the Director of the St George's Academy of Intraoperative and Intensive Care Echocardiography. This is a nonprofit training organization. Echocardiography simulation is one of the teaching techniques used on courses. Inventive Medical provided some free simulators for courses at St George's Hospital. All others authors have nothing to disclose with regard to commercial support.

\section{References}

1. Memtsoudis SG, Rosenberger P, Loffler M, Eltzschig HK, Mizuguchi A, Shernan SK, et al. The usefulness of transoesophageal echocardiography during intraoperative cardiac arrest in noncardiac surgery. Anesth Analg. 2006;102: 1653-7. 
2. Fletcher SN, Grounds RN. Critical care echocardiography: cleared for take up. Br J Anaesth. 2012;109:490-2.

3. Barber RL, Fletcher SN. A review of echocardiography in anaesthetic and peri-operative practice. Part 1: impact and utility. Anaesthesia. 2014;69:764-76.

4. Sharma V, Fletcher SN. A review of echocardiography in anaesthetic and peri-operative practice. Part 2: training and accreditation. Anaesthesia. 2014; 69:919-27.

5. American Society of Anesthesiologists and Society of Cardiovascular Anesthesiologists Task Force on Transesophageal Echocardiography. Practice guidelines for perioperative transesophageal echocardiography: an updated report. Anesthesiology. 2010;112:1084-96.

6. Reeves ST, Finley AC, Skubas NJ, Swaminathan M, Whitley WS, Glas KE, et al. Basic perioperative transesophageal echocardiography examination: a consensus statement of the American Society of Echocardiography and the Society of Cardiovascular Anesthesiologists. J Am Soc Echocardiogr. 2013;26:443-56.

7. Intercollegiate Surgical Curriculum Programme. Available at: https://www.iscp. ac.uk/documents/syllabus_CT_2013.pdf. Accessed April 25, 2014

8. Skinner H, Morgan-Hughes N, Swanevelder J, More R. Accreditation in transoesophageal echocardiography in the UK: the initial experience. $\mathrm{Br} J$ Anaesth. 2012;109:487-90.

9. Sharma V, Chamos C, Valencia O, Meineri M, Fletcher SN. The impact of internet and simulation-based training on transoesophageal echocardiography learning in anaesthetic trainees: a prospective randomised study. Anaesthesia. 2013;68:621-7

10. Okuda Y, Bryson EO, DeMaria S Jr, Jacobson L, Quinones J, Shen B, et al. The utility of simulation in medical education: what is the evidence? Mt Sinai J Med. 2009;76:330-43.

11. Clau-Terre F, Sharma V, Cholley B, Gonzalez-Alujas T, Galiñanes M, Evangelista A, et al. Can simulation help to answer the demand for echocardiography education? Anesthesiology. 2014;120:32-41.
12. Bose R, Matyal R, Panzica P, Karthik S, Subramaniam B, Pawlowski J, et al Transesophageal echocardiography simulator: a new learning tool. $J$ Cardiothorac Vasc Anesth. 2009;23:544-8.

13. Bose RR, Matyal R, Warraich HJ, Summers J, Subramaniam B, Mitchell J, et al. Utility of a transesophageal echocardiographic simulator as a teaching tool. $J$ Cardiothorac Vasc Anesth. 2011;25:212-5.

14. Sohmer B, Hudson C, Hudson J, Posner GD, Naik V. Transesophageal echocardiography simulation is an effective tool in teaching psychomotor skills to novice echocardiographers. Can J Anaesth. 2014;61:235-41.

15. Damp J, Anthony R, Davidson MA, Mendes L. Effects of transesophageal echocardiography simulator training on learning and performance in cardiovascular medicine fellows. J Am Soc Echocardiogr. 2013;26:1450-6.

16. Ferrero N, Bortsov A, Arora H, Martinelli SM, Kolarczyk LM, Teeter EC, et al Simulator training enhances resident performance in transesophageal echocardiography. Anesthesiology. 2014;120:149-59.

17. Shanewise JS, Cheung AT, Aronson S, Stewart WJ, Weiss RL, Mark JB, et al ASE/SCA guidelines for performing a comprehensive intraoperative multiplane transesophageal echocardiography examination: recommendations of the American Society of Echocardiography Council for Intraoperative Echocardiography and the Society of Cardiovascular Anesthesiologists Task Force for Certification in Perioperative Transesophageal Echocardiography. Anesth Analg. 1999;89:870-84.

18. Ogilvie E, Vlachou A, Edsell M, Fletcher SN, Valencia O, Meineri M, et al Simulation-based teaching versus point-of-care teaching for identification of basic transesophageal echocardiography views: a prospective randomised study. Anaesthesia. 2015;70:330-5.

Key Words: computer simulation, medical education, transesophageal echocardiography

Readers who found these articles interesting may also like to read the following papers found in recent and future issues of our sister publications, Seminars in Thoracic and Cardiovascular Surgery and Operative Techniques in Thoracic and Cardiovascular Surgery!

\section{Cardiothoracic Surgical Education and Training}

News and Views: Integrated Surgical Residency Initiative: Implications For Cardiothoracic Surgery-John Ikonomidis and Editorial Commentary-Ara Vaporciyan. Semin Thorac Cardiovasc Surg. 2014 Spring;26(1):14-26.

Discussion in Cardiothoracic Treatment and Care: Manpower-Richard Shemin, David Fullerton, John Ikonomidis, John Mayer, Cameron Wright. Semin Thorac Cardiovasc Surg. 2014; Autumn; 26(3):255-271. 